very useful for heating or evaporating solutions, where a temperature higher than that attainable on a water bath is necessary and where it is necessary to avoid local overheating, which is so liable to occur if a dish rests directly on the hot plate. A set of ordinary water-bath rings may be substituted for the watch-glass cover, thus enabling any size of evaporating dish or casserole to be heated uniformly and to any desired temperature up to $375^{\circ}$.

Chemical I abotatory,

University of Michigan, Dec. 27, 1906.

\title{
THE INFLUENCE OF BASIC LEAD ACETATE ON THE ROTATION OF SUCROSE IN WATER SOLUTION.
}

BY FREDERICK BATES AND J. C. BLAKE.

Received Jantuaty 7,1907 .

In the polarimetric estimation of sucrose in raw sugars an indefinite amount of basic lead acetate in solution is usually added to the sugar flask, before making up to volume, in order to precipitate the impurities more or less completely and thus clarify the sugar solution sufficiently for reading on the polariscope. The use of this reagent is almost universal, and it is, indeed, so powerful a clarifying reagent that the question as to whether it can be used in precision work on raw sugars is of the first importance. Excepting the error due to the volume of the precipitate which is formed by the reaction it is usually considered by commercial chemists that the results are not affected by the basic acetate.

The evidence obtainable from the literature as to the effect of basic lead acetate on the polarization of sucrose is conflicting and very unsatisfactory, the results ustually being expressed in tenths of a per cent, with no indications that attention was paid to numerous minor sources of error. Von Lippmann's ${ }^{1}$ conclusion, derived from the literature, is that basic lead acetate exerts no influence on the rotation of sucrose in water solution. We have found this conclusion to be in error. Its great influence on the specific rotation of levulose has long been known. Nevertheless, because of the relatively large amount of sucrose present in raw sugars as compared to the amount of levulose present, the effect of the basic lead acetate on the sucrose, though relatively less than its effect on levulose, still retains an equal importance.

The effect of basic lead acetate on the specific rotation of sucrose was overlooked by the early investigators because of the comparatively crude polarizing aparatus and methods used, and also because the temperature coefficient of pure sugar solutions was little known or entirely neglected.

${ }^{1}$ Chemie der Zuckerarten 2 , I 85. 
A few preliminary experiments soon demonstrated that it would not be safe to carry out the present investigation on an ordinary commercial saccharimeter alone. An accuracy of $0.02^{\circ} \mathrm{V}$ was desired. The greatest inherent difficulty in obtaining this accuracy lay in the polarizing appa-

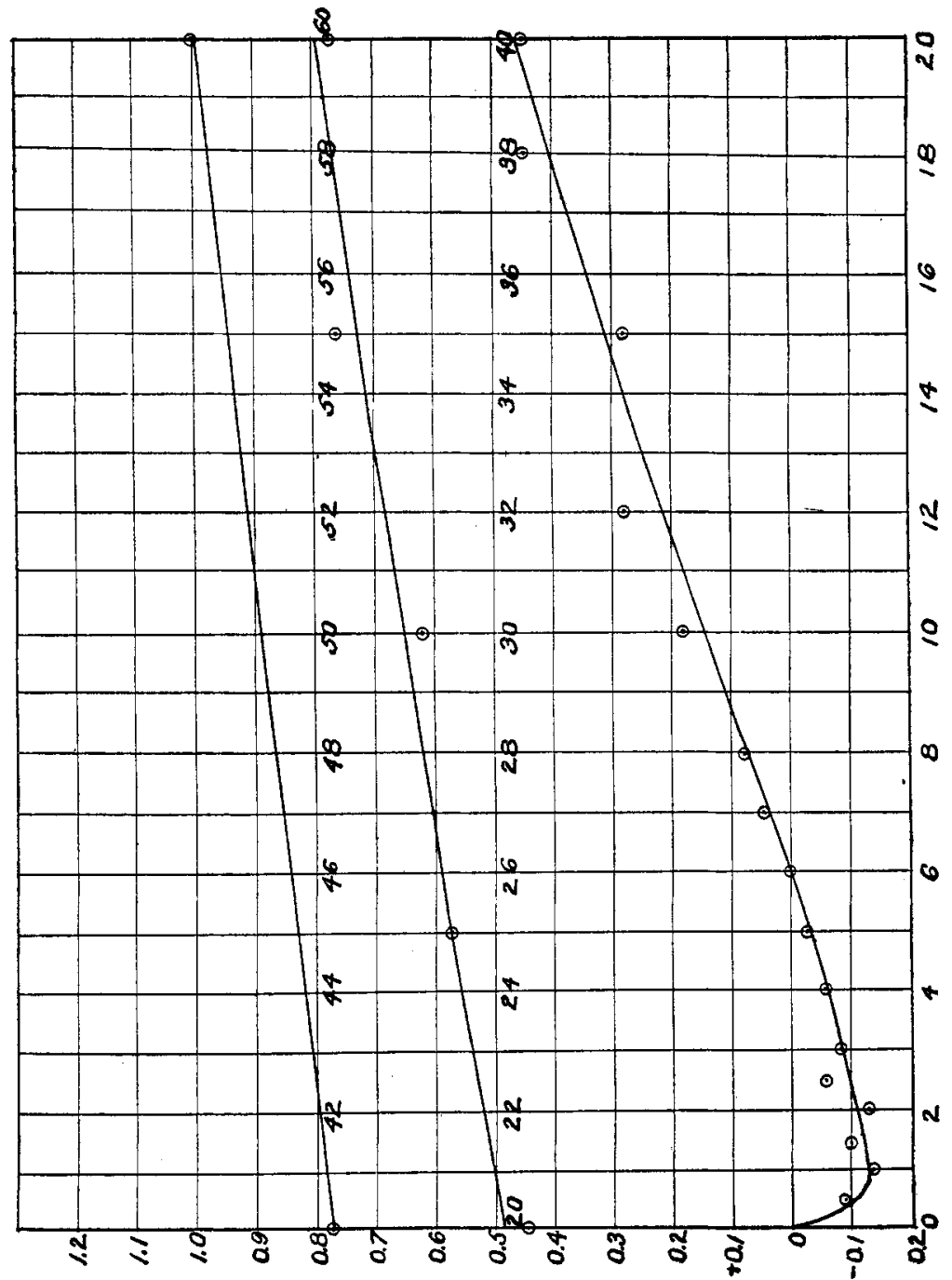

ratus. It was overcome by improving a Josef and Jan Fric double quartz wedge compensation saccharimeter. The instrument has glass scales and verniers, the light reaching the eye of the observer being transmitted and not reflected. As a result the effect of temperature changes on the length 
of the scale is very small, and the defuition of the ruling is such that the scale can readily be interpolated to a hundredth of a degree Ventzke. The polarizing nicol was removed and a first class Lippich half-shade system substituted. The light was passed through $1.5 \mathrm{~cm}$ of a six per cent potassium bichromate solution before it entered the polarizing system. An improved electric lamp of great intensity was used as the source of light, thereby permitting of the use of a polarizing angle of $4.33^{\circ}$ (circular). This angle is too small to be utilized with the ordinary mechanims for shifting the wedge. The instrument was accordingly fitted with an auxiliary screw adjustment by means of which the accurate settings were made. With the improvements as indicated above the sensitiveness of the saccharimeter was such that a difference in rotation of $0.02^{\circ}$ Ventzke could be detected with certainty by the observer without making a series of observations ; differences of that magnitude being sufficient to produce a directly discernable change in the intensities of the two halves of the field. In table I are given ten consecutive readings on a tube of solution in this instrument. They were selected at random.

\section{TABLE I.}

Consecutive readings on Sugar Tube

Degrees ventzke

99.98

I00.00

99.99

99.99

99.99

99.99

99.98

99.98

99.98

99.98

The surfaces of the wedges were sufficientiy plane to justify this degree of refinement. 'The length used where the differences in rotation were measured corresponded to not more than I. $I^{\circ} \mathrm{V}$ of the scale. The 100 cc. flasks were especially made for this work. The necks had an internal diameter of Io $\mathrm{mm}$. The volumes were known to within o. I cc. All temperatures were read on thermometers graduated to $0.0 \mathrm{I}^{\circ}$. The tubes were $200 \mathrm{~mm}$ in length enlarged at one end. From the middle of the tube and at right angles to its length projected a glass tube $2.5 \mathrm{~cm}$. Through this a thermometer was passed, its buib being immersed in the solution. A rubber washer closed the space between the tube and thermometer. All the apparatus used was standardized for a temperature of $20 .^{\circ}$.

The sugar used in these experiments was the best grade of commercial sugar. Two samples were carefully prepared from sugar purchased at different times. Precautions were taken to have each sample as thor- 
oughly mixed as possible. After much consideration it was concluded that practically nothing would be gained by using chemically pure sucrose. The procuring of the large amount required would have been a serious difficulty. The basic lead acetate solution was prepared either by the action of the neutral acetate in solution on lead oxide at the boiling temperature, or by simply dissolving a high grade product in water. The solution was made up to a density of 1.25 at $15^{\circ}$, and when titrated with acid gave approximately the theoretical amount of dissolved lead oxide.

It is desirable that investigations of this character be carried out in a room whose temperature can be maintained at the standard temperature of $20^{\circ}$. Such a room was not available at the time these experiments were made, but the greatest care was taken to correct the results for the variations introduced by temperature changes, although the errors that might have been introduced by these variations were very small. The temperature coefficient of a pure sucrose solution containing 26 grams of sucrose in 100 true cubic centimeters when made up and polarized at a temperature other than the standarization temperature of the apparatus has been redetermined in this laboratory for both a Schmidt and Haensch polariscope and the improved Fric instrument described above. From these results, not yet published, and the fairly concordant results previously obtained by others on the Schmidt and Haensch instrument, the following values were adopted for making the various temperature corrections for variations from $20^{\circ}$, not exceeding 5 degrees, except in two instances.

I. Coefficient of cubical expansion of standard sugar solution at $23^{\circ} \pm$ $2^{\circ}=0.029^{\circ} \mathrm{V}$.

2. Temperature coefficient of standard sugar solution made up at $23^{\circ}$ $\doteq 2^{\circ}$ read with glass tubes on a quartz wedge polariscope at $23^{\circ} \pm 2^{\circ}=$ $0.03 \mathrm{I}^{\circ} \mathrm{V}$.

Although the coefficient of expansion of a standard sugar solution varies with the temperature, yet the value here given is sufficiently accurate for our purpose, since the difference between the temperature of filling the flasks and the polarizing temperature was always less than $2^{\circ}$. Hence the error could hardly reach $0.0 \mathrm{I}^{\circ} \mathrm{V}$.

The method of procedure finally adopted was as follows :

(I) $26.048 \pm 0.0003 \mathrm{~g}$. sugar were weighed out, all the determinations for one day being weighed out within a short interval of time. The error due to weighing was, therefore, $\pm 0.00 I^{\circ} \mathrm{V}$.

(2) The sugar was washed into a freshly-cleaned flask with ordinary distilled water and dissolved.

(3) The basic lead acetate solution was then added, usually resulting in a turbidity. This turbidity is especially troublesome for amounts of 
basic lead acetate solution in the neighborhood of three to four cubic centimeters. It can not be filtered out with paper filters.

(4) One or two cubic centimeters of a lead sulphate cream of constant composition were then added to the solution to faciliate filtration.

(5) The solution was then made up to the mark by immersing the flask in a water bath, the temperature of filling being determined by means of another flask filled with water similarly placed. The time thus required insured the complete drainage of the water in the neck of the flask. The solution was made as nearly homogeneous as possible before filling, to avoid errors due to contraction on dilution.

(6) The solution was then thoroughly shaken up and poured into a barium sulphate "Faltenfilter," Schleicher and Schüll No. 605. The filtrate was usually clear at the start. The portion used for polariscopic reading was taken after the liquid had been on the filter almost one minute.

(7) The polariscope tubes were filled by simple pouring and the solutions polarized on the apparatus described above. The readings were made in a room ( $1.4 \mathrm{~m} \times 2.1 \mathrm{~m} \mathrm{x} 4 \mathrm{~m}$ ) especially constructed for work of this nature. It was made by partitioning off one corner of the laboratory, and permitted the exclusion of all extraneous light. It was maintained at practically the same temperature as the laboratory by means of air circulation controlled by an electric fan.

The temperature corrections were applied as follows:

The average of the temperature of the solution and the temperature read on a thermometer hanging with its bulb in contact with the polariscope was taken as the polarizing temperature. The difference between this temperature and twenty degrees added to the difference between the polarizing temperature and the temperature of filling (which was always the smaller value) was multiplied by 0.03 and added to the polariscopic reading. The volume of the lead sulphate added was then subtracted and the corrections for the flasks and polariscopic tubes at $20^{\circ}$ applied.

The lead sulphate added to facilitate filtration had no effect on the polariscopic reading after allowance was made for the volume of the sulphate. This is shown by the data in Table II, which contains six separate determinations each in duplicate, three of which contained $2 \mathrm{cc}$. of lead sulphate and three contained no lead sulphate. The volume of the sulphate in I cc. of the cream was $0.045 \mathrm{cc}$. After the data given in Table III had been obtained it was found that the turbidity could be avoided by using water free from carbonates and preventing too great a dilution of portions of the lead subacetate-sugar solution in filling the flask to the mark. Accordingly much of the work was repeated without 
the use of any lead sulphate. The results were in complete agreement with those given in Table III.

TABLE II.

Effect of 2 cc. $\mathrm{PbSO}_{4}$

\begin{tabular}{crrrr} 
Date & \multicolumn{3}{c}{$\begin{array}{c}\text { With } \text { PbsO }_{4} \\
\text { Bates }\end{array}$} \\
May 2 & $\ldots$ & $\ldots$ & 99.90 \\
June 12 & $\ldots$ & $\ldots$ & .89 & .895 \\
June 28 & $\ldots$ & $\ldots$ & .895
\end{tabular}

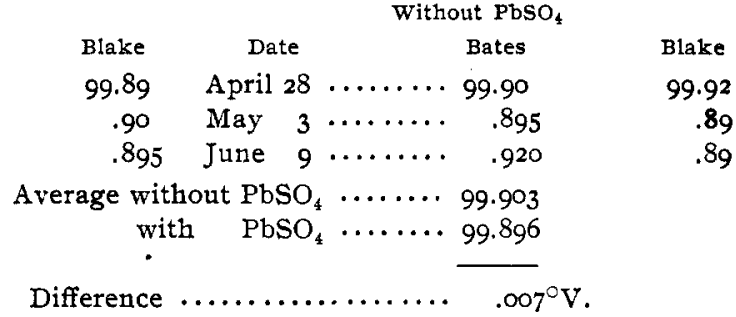

The temperature coefficient of the normal sugar solution is not changed by the addition of basic lead acetate, if at all, sufficiently to effect the results obtained by using the coefficient for a pure sugar solution. This was determined by measuring the temperature coefficient of a normal sugar solution containing $20 \mathrm{cc}$. of the basic lead acetate solution. The range of temperature was $14^{\circ}$. The result obtained was in complete agreement with the value given by Schonröck who found ${ }^{1}$ for the normal stugar solution

$$
a_{D}^{20}=a_{D}^{t}+a(0.09046 \mathrm{I})(t-20)
$$

for all wave lengths. The coefficient $0.046 \mathrm{I}(0.00046 \mathrm{I} \times \mathrm{I} 00=0.046 \mathrm{I})$ was measured in preference to the coefficient $0.03 \mathrm{I}$, because while both involve the temperature coefficient of the specific rotation, which is the only thing that would be appreciably affected by the presence of basic lead acetate, the 0.031 involves the temperature coefficient of the instrument and $0.046 \mathrm{I}$ does not.

The data from the measurements are given in Table III. The first and second columns give the dates on which the different polarizations were made. The third column contains the number of $\mathrm{cc}$. of basic lead acetate added. The differences between the solution containing no basic lead acetate and one containing a given amount is given for both observers in the columns headed Sample No. I and Sample No. 2. The + sign indicates that the solution containing the reagent gives the higher polarization and inversely for the - sign. The average of the differences found by both observers is given in the final column. At least five settings were made by each observer on each tube. Of the large number of observations made only three isolated ones, which were obviously in error, have been discarded. Each difference given in the table is the average of two separate determinations made simultaneously, except when otherwise stated.

1 Z. Ver. Deut. Zuck-Ind., 53, 650. 
TABLE IIT.

EFFECT OF BASIC LEAD ACETATE OX NoRMAI, SOLCTIONS.

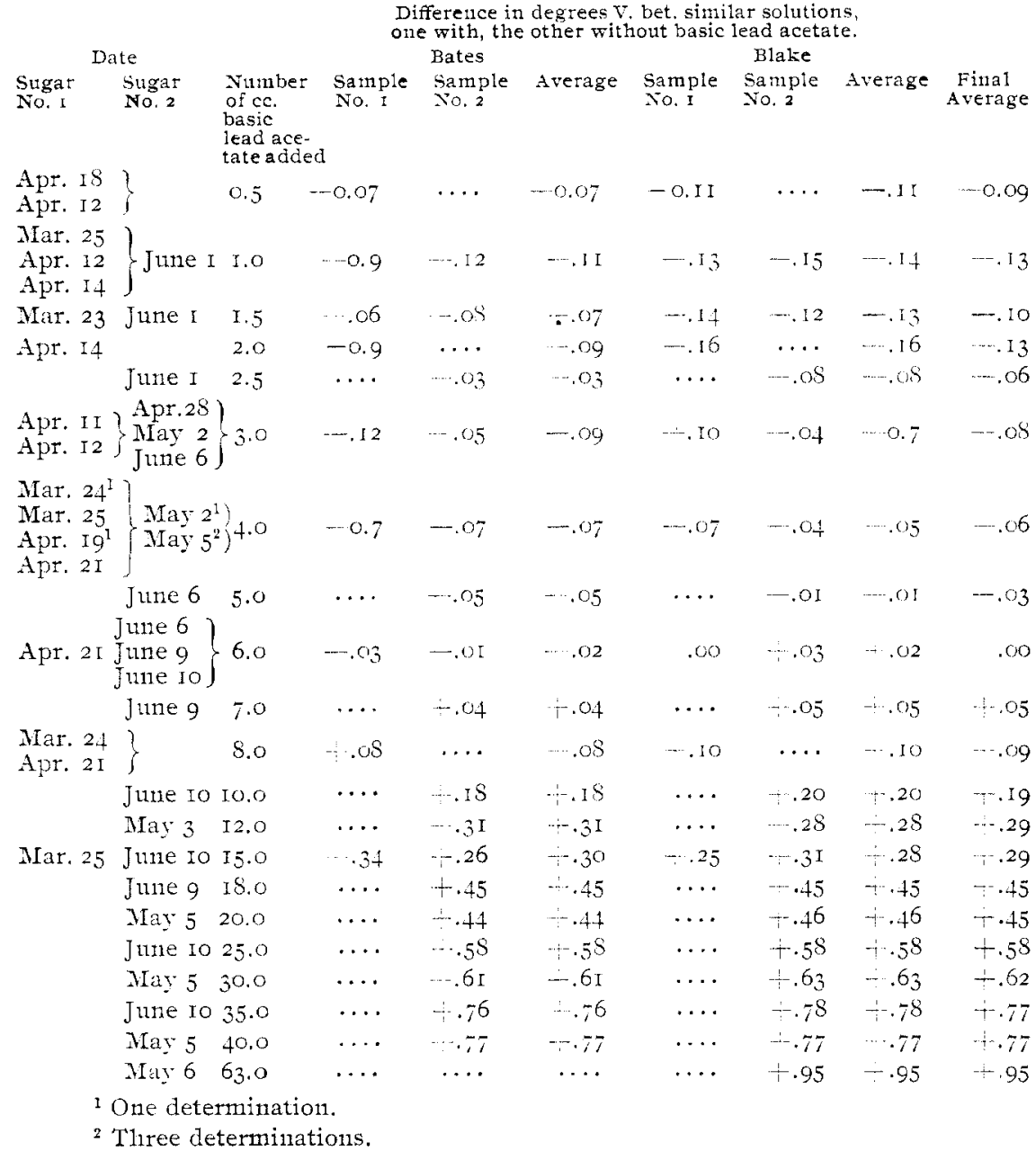

The results seem to be consistent within the limit of accuracy sought $\left(0.02^{\circ} \mathrm{V}\right)$. Even when considerable variations occurred, as with $2.5 \mathrm{cc}$ basic lead acetate acting on sugar No. 2, the average value falls pretty close to the smooth curve from the results. The constancy of the blank determinations made on sugar No. 2 was remarkable. The value of sugar No. I, on the contrary, continually decreased, the total change from March 23 to May 25 being $0.15^{\circ} \mathrm{V}$. The value of sugar No. 2 was, therefore, assumed constant for days on which no blank deternination was made; whereas values obtaine? by interpolation were used under similar circumstances for sugar No. I. The changes in No. I were so gradual 
and extended over such a long time interval that no error larger than $0.0 I^{\circ} \mathrm{V}$ could have been introduced by the interpolation. The variations between the two observers are of opposite sign on the blank determinations of the two sugars, and are, therefore, accidental. The data were plotted graphically. Owing to the length of the curve it was plotted in three sections all on the same scale. The ordinates, in degrees $\mathrm{V}$, are the differences between the polarizations of two solutions, one of which contained basic lead acetate, the other none. The zero point represents the blank solution. When the polarization given by the basic lead acetate solution is the larger the difference is given the + sign and inversely for the - sign. The abscissas are the volumes of basic lead acetate added, expressed in cubic centimeters.

The curve shows beyond doubt that under the given circumstances basic lead acetate first causes a lowering of the polariscopic reading of sugar in the solution amounting to more than $0 . r^{\circ} \mathrm{V}$ for normal concentration, and that further addition of the same reagent causes a continuous rise in the polarization up to the limit $(63 \mathrm{cc})$ investigated. It will be observed that when about $6 \mathrm{cc}$ of basic lead acetate are added the polarization is not affected, the curve crossing the axis at this point.

This effect of basic lead acetate on the polarization of sugar seems to be due to the formation of soluble lead saccharates having specific rotations different from that of sugar. Lead saccharates having properties which would account for the general nature of the curve have already been studied in connection with this investigation.

The values in the table show clearly the error that may be introduced in polarizations where relatively large amounts of the basic lead acetate are used. The depression in the curve, corresponding to small amounts of the reagent, is of special significance in the polarimetric estimation of sucrose in raw sugars and is of sufficient magnitude to place it along with the errors introduced by the volume of the precipitate, the temperature coefficient, and the presence of invert sugar and other impurities.

BUREAU OF STANDARDS,

WASHINGTON, D. C.

[CONTRIBCTION FROM THE BUREAU OF CHEMISTRY, U. S. THEPARTMENT OF AGRICLITURE.]

\section{THE USE OF CARBON BISULPHIDE IN THE ESTIMATION OF SALICYLIC ACID IN WINE.}

BY W. I. Dtbors.

In determining salicylic acid in wines a great deal of trouble is exnerienced with tannin, coloring matter and such bodies, which dissolve in 